\title{
CD73 promotes colitis-associated tumorigenesis in mice
}

\author{
XUAN-HUI LIU ${ }^{1,2^{*}}$, XIAN-RUI WU ${ }^{1-3^{*}}$, NAN LAN ${ }^{1,2}$, XIAO-BIN ZHENG $^{1,2}$, CHI ZHOU $^{1,2}$, TUO HU $^{1,2}$, \\ YU-FENG CHEN $^{1,2}$, ZE-RONG CAI $^{1,2}$, ZE-XIAN CHEN $^{1,2}$, PING LAN $^{1,2}$ and XIAO-JIAN WU ${ }^{1,2}$
}

\begin{abstract}
Departments of ${ }^{1}$ Colorectal Surgery and ${ }^{2}$ Guangdong Provincial Key Laboratory of Colorectal and Pelvic Floor Diseases, The Sixth Affiliated Hospital of Sun Yat-sen University; ${ }^{3}$ Guangzhou Regenerative Medicine and Health Guangdong Laboratory, Guangzhou, Guangdong 510655, P.R. China
\end{abstract}

Received June 26, 2019; Accepted April 29, 2020

DOI: $10.3892 / \mathrm{ol} .2020 .11670$

\begin{abstract}
Patients with inflammatory bowel disease (IBD) are at a higher risk of developing colitis-associated colorectal cancer. The aim of the present study was to investigate the role of CD73 in IBD-associated tumorigenesis. A mouse model of colitis-associated tumorigenesis (CAT) induced by azoxymethane and dextran sulfate sodium was successfully constructed. Model mice were injected with CD73 inhibitor or adenosine receptor agonist. Colon length, body weight loss and tumor formation were assessed macroscopically. Inflammatory cytokine measurement and RNA sequencing on colon tissues were performed. Inhibition of CD73 by adenosine 5 '-( $\alpha, \beta$-methylene) diphosphate (APCP) suppressed the severity of CAT with attenuated weight loss, longer colons, lower tumor number and smaller tumor size compared with the model group. Activation of adenosine receptors using 1-(6-amino-9H-purin-9-yl)-1-deoxy-N-ethyl- $\beta$-D-ribofuranur onamide (NECA) exacerbated CAT. Histological assessment indicated that inhibition of CD73 reduced, while activation of adenosine receptors exacerbated, the histological damage of the colon. Increased expression of pro-inflammatory cytokines (tumor necrosis factor- $\alpha$ and interleukin-6) in colonic tissue was detected in the NECA group. According to RNA
\end{abstract}

Correspondence to: Dr Ping Lan or Dr Xiao-Jian Wu, Department of Colorectal Surgery, The Sixth Affiliated Hospital of Sun Yat-sen University, 26 Yuancun Erheng Road, Guangzhou, Guangdong 510655, P.R. China

E-mail: lanping@mail.sysu.edu.cn

E-mail:wuxjian@mail.sysu.edu.cn

*Contributed equally

Abbreviations: IBD, inflammatory bowel disease; CRC, colorectal cancer; CAT, colitis-associated tumorigenesis; APCP, adenosine 5'-( $\alpha, \beta$-methylene) diphosphate; NECA, 1-(6-amino-9H-purin-9-yl)-1-deoxy-N-ethyl- $\beta$-D-ribofuranuronamide; $\mathrm{AOM}$, azoxymethane; DSS, dextran sulfate sodium; RT-qPCR, reverse transcription-quantitative PCR; HE, hematoxylin and eosin; IHC, immunohistochemistry; SEM, standard error mean

Key words: IBD, colitis-associated tumorigenesis, CD73 sequencing results, potential oncogenes such as arachidonate 15-lipoxygenase (ALOX15), Bcl-2-like protein 15 (Bcl2115) and $\mathrm{N}$-acetylaspartate synthetase (Nat8l) were downregulated in the APCP group and upregulated in the NECA group compared with the model group. Therefore, inhibition of CD73 attenuated IBD-associated tumorigenesis, while activation of adenosine receptors exacerbated tumorigenesis in a C57BL/6J mouse model. This effect may be associated with the expression of pro-inflammatory cytokines and the regulation of ALOX15, Bcl2115 and Nat81.

\section{Introduction}

Inflammatory bowel disease (IBD), including Crohn's disease and ulcerative colitis, is a chronic, relapsing inflammatory disorder of the digestive tract $(1,2)$. Chronic inflammation is considered to play an important role in the development of cancer $(3,4)$. Colorectal cancer (CRC) is the most serious complication of IBD, and patients with IBD are at a significantly increased risk of developing CRC compared with the general population, worldwide $(5,6)$. This may be a representative example reflecting the association between chronic inflammation and tumorigenesis. Lutgens et al (7) demonstrated that the pooled standardized incidence ratio of CRC in all patients with IBD in worldwide population-based studies was 1.7 , while the cumulative risk for CRC in IBD patients was 1,2 and $5 \%$ at 10,20 and $>20$ years of disease duration, respectively.

CD73, also referred to as ecto-5'-nucleotidase, is a membrane-bound glycoprotein, the primary function of which is to hydrolyze extracellular nucleoside monophosphates into bioactive nucleoside intermediates, leading to the generation of extracellular adenosine (8). Adenosine has multiple functions aimed at maintaining tissue homeostasis, and mediates its immunosuppressive effects mainly via $\mathrm{A} 2 \mathrm{~A}$ and $\mathrm{A} 2 \mathrm{~B}$ receptors (9). CD73 is upregulated in several types of cancer and increasing evidence suggested that CD73 plays a crucial role in the control of tumor progression (10-12). It was demonstrated that inhibition of CD73 activity or CD73 knockdown on tumor cells inhibited tumor growth by enhancing the antitumor T-cell response $(13,14)$. By using CD73-deficient mice, it was demonstrated that CD73 on hematopoietic cells (including Foxp $3^{+}$Treg cells) impairs the antitumor T-cell-mediated immune response. These effects are attributed to the regulation of extracellular adenosine generated by CD73 within the 
tumor microenvironment $(15,16)$. Additionally, CD73 research on IBD revealed that transfer of $\mathrm{CD}^{2} 3^{+} \mathrm{B}$ cells to $\mathrm{CD} 73^{-1}$ mice decreased the severity of colitis, suggesting that B-cell CD73/CD39/adenosine can modulate dextran sulfate sodium (DSS)-induced colitis (17).

The understanding of the role of CD73 in tumor initiation in patients with IBD remains limited (11). The aim of the present study was to determine the role of CD73 in IBD-associated tumorigenesis in a mouse model by using the CD73 inhibitor adenosine 5 '-( $\alpha, \beta$-methylene) diphosphate (APCP) and the non-selective adenosine receptor agonist 1-(6-amino-9H-purin -9-yl)-1-deoxy-N-ethyl- $\beta$-D-ribofuranuronamide (NECA).

\section{Materials and methods}

Mice. A total of 39 female C57BL/6 mice (age, 6-8 weeks; weight, $\sim 20 \mathrm{~g}$ ) were obtained from the Laboratory Animal Center of Sun Yat-sen University (Guangzhou, China). The mice were kept in a special pathogen-free facility with free access to drinking water and a pellet-based diet, and were quarantined for 7 days prior to the experiment. They were maintained at acontrolled temperature $\left(22 \pm 1^{\circ} \mathrm{C}\right)$, humidity $(50-70 \%)$ and a $12 \mathrm{~h}$ light/dark cycle. The experimental protocol was approved by the Ethics Committee of Sun Yat-sen University (approv al no. SYSU-IACUC-2020-B0038). All animal studies were conducted with the approval of the Institutional Animal Care and Use Committee of Sun Yat-sen University.

Reagents. Azoxymethane (AOM), APCP and NECA were purchased from Sigma-Aldrich; Merck-KGaA. DSS was purchased from MP Biomedicals, LLC.

Animal model induction and treatment. The C57BL/6 mice were divided into four groups (6 mice in the negative control group and 11 mice per experimental group), including the negative control group (receiving no AOM/DSS or other treatment), the model control group (receiving AOM/DSS and PBS treatment), the CD73 inhibitor group (APCP group; receiving AOM/DSS and APCP) and the adenosine receptor agonist group (NECA group; receiving AOM/DSS and NECA).

AOM and DSS were used to induce colitis-associated tumorigenesis (CAT) in the mice. Briefly, the mice were injected intraperitoneally with a single dose $(10 \mathrm{mg} / \mathrm{kg})$ of AMO, followed by three cycles of DSS, with each cycle consisting of 1 week of $2 \%$ DSS in the drinking water and 2 weeks of normal drinking water. While drinking 2\% DSS water, mice in the three experimental groups were injected intraperitoneally with APCP $(80 \mu \mathrm{g}, 0.2 \mathrm{ml})(18,19)$ or NECA $(24.66 \mu \mathrm{g}, 0.2 \mathrm{ml})(20)$ or PBS $(0.2 \mathrm{ml})$ every other day. Mice in the control group were intraperitoneally injected with PBS and received pure water for 9 weeks. No measurable toxicity was observed after using the indicated doses of APCP and NECA.

The mice were monitored for body weight, stool consistency and the presence of blood in the excreta on days $1,3,5$ and 7 when drinking water with $2 \%$ DSS, and every 3 days when drinking normal water in each cycle. At the end of week 9 , the mice were sacrificed by cervical dislocation. Colon length (from the ileocecal junction to the anal verge) were measured. Subsequently, the colon was incised longitudinally and macroscopically visible tumor number per mouse, tumor diameter and tumor burden (determined by summation of total tumor volume) were measured with a caliper. Segments of the distal colon were fixed in $10 \%$ neutral buffered formalin (pH7.4) at room temperature for at least $24 \mathrm{~h}$ for subsequent paraffine embedding, or kept in RNAlater ${ }^{\mathrm{TM}}$ stabilization solution (Ambion; Thermo Fisher Scientific, Inc.) as tissue samples for further analysis. Details of the humane endpoints in our mouse model are shown in Table SI. Humane endpoints in the AOM/DSS-induced CAT mouse model experiment suggested to have a total score $\geq 8$ or with the highest point in at least two individual items or with non-CAT-associated symptoms, such as skin lesion and allergy, based on the humane endpoints scoring system (21). After last record, analgesic administration and euthanasia were carried out for animals that reached the humane endpoints. Anticipated weight loss of over $20 \%$ in the NECA group, which did not reach the humane endpoints was considered and approved by the ethics committee.

Histopathological evaluation. Sections (4- $\mu \mathrm{m})$ of formalin-fixed (10\% neutral buffered formalin at room temperature for at least $24 \mathrm{~h}$ ), paraffin-embedded tissues were stained with hematoxylin for 2-3 min and eosin for 1-2 min (HE) at room temperature to evaluate the severity of inflammation using a DMI400B inverted microscope (magnification x10; Leica Microsystems $\mathrm{GmbH}$ ). Colitis was scored in a blinded manner as previously described (22), with a combined score for tissue injury (score, 0-3) and infiltration of inflammatory cells (score, 0-3). Briefly, for tissue injury, $0=$ normal colonic mucosa; 1 = discrete lymphoepithelial lesions; 2 , surface mucosal erosion or focal ulceration and 3, extensive mucosal damage and extension into deeper layers. For infiltration of inflammatory cells: 0, occasional presence of inflammatory cells in the lamina propria; 1 , increasing number of inflammatory cells in the lamina propria; 2 , inflammatory cells extending into the submucosa; and 3 , transmural extension of the infiltration. The histological score was defined as the sum of the two aforementioned parameters (score, 0-6).

Reverse transcription-quantitative PCR (RT-qPCR) analysis. Total RNA was extracted from colonic segments using TRIzol $^{\circledR}$ reagent (Invitrogen; Thermo Fisher Scientific, Inc.). RNA quality and concentration were then assessed using a NanoDrop ND-2000 spectrophotometer (Thermo Fisher Scientific, Inc.). RNA (1 $\mu \mathrm{g})$ was then reverse-transcribed using the ReverTra Ace qPCR RT kit (cat. no. FSQ-101; Toyobo Life Science), according to the manufacturer's protocol. qPCR was performed using SYBR Green Realtime PCR master mix (cat. no. QPK-201; Toyobo Life Science) on an Applied Biosystems 7500 Real-time PCR system (Applied Biosystems; Thermo Fisher Scientific, Inc.). The following thermocycling conditions were used: Initial denaturation at $95^{\circ} \mathrm{C}$ for $10 \mathrm{~min}$; 40 cycles of denaturation at $95^{\circ} \mathrm{C}$ for $10 \mathrm{sec}$, annealing at $60^{\circ} \mathrm{C}$ for $20 \mathrm{sec}$ and elongation at $72^{\circ} \mathrm{C}$ for $30 \mathrm{sec}$; and a final extension at $72^{\circ} \mathrm{C}$ for $7 \mathrm{~min}$. Levels of tumor necrosis factor (TNF)- $\alpha$, TNF- $\beta$, interleukin (IL)-10 and IL-6 were measured. All reactions were performed in triplicate, with 3 samples from different groups. The quantification of target mRNA was normalized against GAPDH. The relative expression levels of each gene were calculated and normalized using the $2^{-\Delta \Delta C q}$ method (23). All primer sequences are listed in Table SII. 
Cell culture, transfection and infection. The human colorectal cancer cell lines HCT8 and RKO were purchased from the Cell Bank of Type Culture Collection of the Chinese Academy of Sciences. Cells were routinely grown in DMEM (Gibco; Thermo Fisher Scientific, Inc.) supplemented with $10 \%$ FBS (Gibco; Thermo Fisher Scientific, Inc.) and incubated at $37^{\circ} \mathrm{C}$ in $5 \% \mathrm{CO}_{2}$ and humidified atmosphere. CD73-overexpressed cell lines were generated by lentiviral transduction of a CD73 human cDNA open reading frame clone using a plasmid reformed from pMSCV-EcoRI-BglII-Zeocin $(15 \mu \mathrm{g}, 1 \mu \mathrm{g} / \mu \mathrm{l}$; cat. no. 75088; Addgene, Inc.). The empty vector was used as a control. All plasmids were verified by sequencing. Lipofectamine $^{\mathrm{TM}} 3000$ (Invitrogen; Thermo Fisher Scientific, Inc.) was used for transfection, according to the manufacturer's protocol. Cells were cultured for $48 \mathrm{~h}$ until they reached $>90 \%$ confluence, and subsequently digested using TRIzol reagent (Invitrogen; Thermo Fisher Scientific, Inc.) and collected for RT-qPCR analysis.

RNA sequencing and analysis. RNA samples extracted from colonic segments were collected from the three experimental groups (model, APCP and NECA groups). Library construction and sequencing were performed using the BGISEQ-500 platform of the Beijing Genomic Institution (www.bgitechsolutions.com; BGI Group). The first step in the workflow involved purifying the poly-A-containing mRNA molecules using poly-T oligo-attached magnetic beads. Following purification, the mRNA was fragmented into small pieces using divalent cations under $70^{\circ} \mathrm{C}$. The cleaved RNA fragments were copied into first-strand cDNA using reverse transcriptase and random primers from the SuperScript ${ }^{\mathrm{TM}}$ III First-Strand Synthesis System (Invitrogen; Thermo Fisher Scientific, Inc.). This was followed by second-strand cDNA synthesis using DNA polymerase I and RNase $\mathrm{H}$. These cDNA fragments then underwent addition of a single 'A' base and subsequent ligation of the adapter. The products were then purified and enriched with PCR amplification (Initial denaturation at $94^{\circ} \mathrm{C}$ for $2 \mathrm{~min}$; 30 cycles of denaturation at $98^{\circ} \mathrm{C}$ for $2 \mathrm{sec}$, annealing at $55^{\circ} \mathrm{C}$ for $30 \mathrm{sec}$ and elongation at $68^{\circ} \mathrm{C}$ for $30 \mathrm{sec}$; and a final extension at $68^{\circ} \mathrm{C}$ for $7 \mathrm{~min}$ ). The PCR yield was then quantified by Qubit (Thermo Fisher Scientific, Inc.) and samples were pooled together to create a single-strand DNA (ssDNA) circle, which provided the final library. DNA nanoballs (DNBs) were generated with the ssDNA circle by rolling circle replication to enlarge the fluorescence signals at the sequencing process. The DNBs were loaded into the patterned nanoarrays and single-end reads of $50 \mathrm{bp}$ were read through on the BGISEQ-500 platform for the following data analysis study. For this step, the BGISEQ-500 platform combined DNB-based nanoarrays and stepwise sequencing using the Combinational Probe-Anchor Synthesis Sequencing Method. The significance of the differential expression of genes was defined by the bioinformatics service of BGI according to the combination of the absolute value of $\log _{2}$-ratio $\geq 1$ and false discovery rate $\leq 0.001$. EuKaryotic Orthologous Group functional classification, Gene Ontology (GO) and pathway annotation and enrichment analyses were based on NCBI COG (https://www.ncbi.nlm.nih.gov/COG/), Gene Ontology Database (http://www.geneontology.org/) and Kyoto Encyclopedia of Genes and Genomes (KEGG) pathway database (http://www.genome.jp/kegg/), respectively.
Statistical analysis. Statistical analysis was performed using SPSS version 22.0 (IBM Corp.). Values are expressed as the mean \pm SEM and multiple comparisons were analyzed using one-way ANOVA and Tukey's post hoc test. Discrete data were compared using Kruskal-Wallis test followed by Dunn's multiple comparison test. Two-tailed unpaired Student's t-test was used to compare the relative mRNA expression levels between CD73-overexpressed cell lines and empty vector controls. $\mathrm{P}<0.05$ was considered to indicate a statistically significant difference.

\section{Results}

AOM/DSS-induced CAT model in C57BL/6J mice. To investigate the role of CD73 and its downstream pathway in CAT, the mice in a CAT model induced by AOM/DSS received CD73 inhibitor (APCP) or adenosine receptor agonist (NECA) treatment. The experimental procedure and the effect on body weight loss in the first cycle are shown in Fig. 1A and B.

By the end of the experiment, 6 mice in the control group, 9 mice in the APCP group, 6 mice in the model group and 4 mice in the NECA group remained alive (Fig. 1C). Mice in the model group exhibited a more significant body weight loss compared with the control group, while mice receiving APCP exhibited a significantly lower body weight loss and mice receiving NECA exhibited a higher body weight loss compared with the mice in the model group ( $\mathrm{P}<0.05$; Fig. 1D).

APCP attenuates, while NECA aggravates, AOM/DSS-induced $C A T$. At the end of week 9, the mice were sacrificed and the colon length (from the ileocecal junction to the anal verge) was measured without tension. Subsequently, the colon was incised longitudinally and macroscopically visible tumors were counted and measured (Fig. 2A and C).

Shortening and swelling of the colon was observed in both groups receiving AOM/DSS compared with the control group. However, the colons in the APCP group were significantly longer compared with the model group (68.29 vs. $62.67 \mathrm{~mm}$, respectively; $\mathrm{P}<0.05$; Fig. $2 \mathrm{~B}$ ), while the colons in the NECA group were shorter compared with the model group (57.50 vs. $62.67 \mathrm{~mm}$, respectively; $\mathrm{P}<0.05$; Fig. $2 \mathrm{~B}$ ). After opening the colons, tumors were observed between the mid colon and the distal rectum in the experimental groups, while no tumors were found in the control group. APCP treatment resulted in a significant reduction in the number of tumors per mouse compared with mice in the model group (5.1 vs. 7.7, respectively; $\mathrm{P}<0.05$; Fig. 2D), while NECA treatment significantly increased the number of tumors per mouse compared with mice in the model group (10.3 vs. 7.7, respectively; $\mathrm{P}<0.05$; Fig. 2D). The mean diameter of the existing tumors was also significantly higher in the NECA group $(2.96 \mathrm{~mm}$ in the NECA group vs. $2.18 \mathrm{~mm}$ in the model group; $\mathrm{P}<0.05$ ), while the diameter of tumors of APCP-treated group were smaller compared with the model group (1.85 $\mathrm{mm}$ in the APCP group vs. $2.18 \mathrm{~mm}$ in the model group), although the difference was not statistically significant (Fig. 2E).

APCP decreases, while NECA increases, histological damage and levels of inflammatory cytokines in colonic tissue. Following macroscopic inspection, segments of the distal 


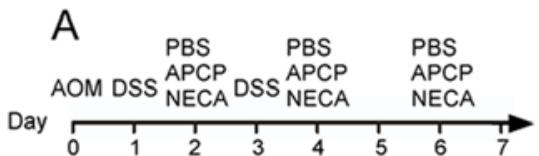

Day

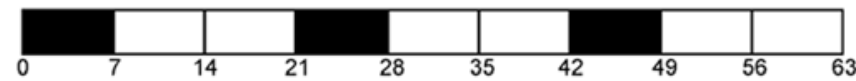

C

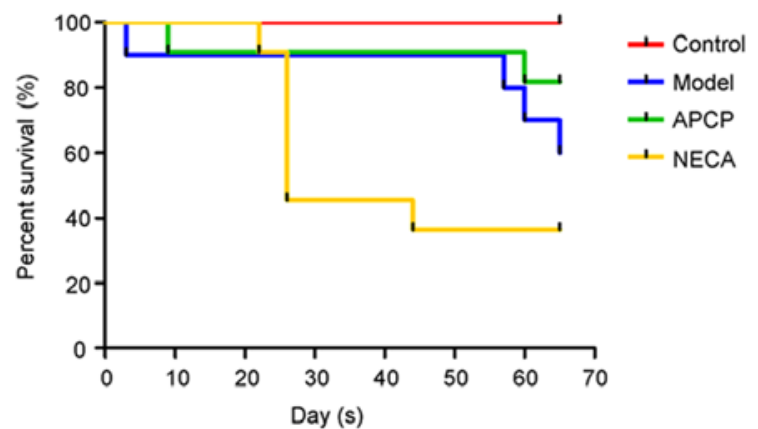

B

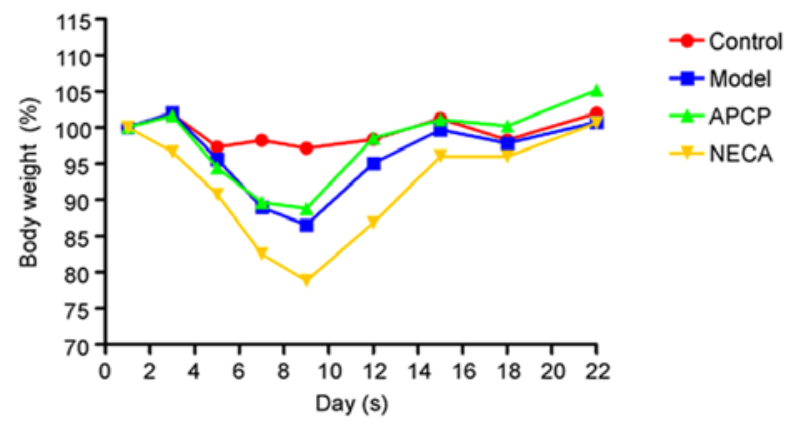

D

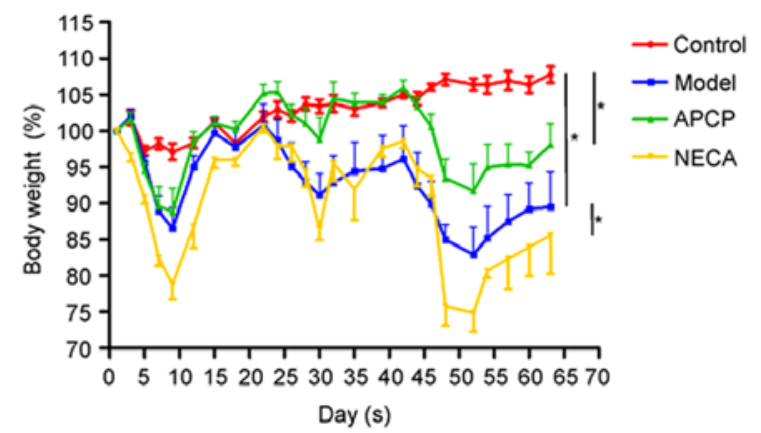

Figure 1. AOM/DSS-induced CAT model in C57BL/6J mice. (A) Experimental protocol of the CAT model. Black box indicate the week of $2 \%$ DSS in the drinking water while white box indicate the week of normal drinking water. Mice in the control group were intraperitoneally injected with PBS and received pure water for 9 weeks. (B) Changes in mouse body weight in the first cycle. (C) A total of 6 mice in the control group, 9 mice in the APCP group, 6 mice in the model group and 4 mice in the NECA group survived during the period of experiment. (D) Mouse body weight changes during the entire experimental period. APCP treatment ameliorated and NECA treatment aggravated weight loss compared with PBS treatment in the experimental groups. Values are expressed as the mean \pm SEM. "P $<0.05$. AOM, azoxymethane; DSS, dextran sulfate sodium; CAT, colitis-associated tumorigenesis; APCP, adenosine 5'-( $\alpha, \beta$-methylene) diphosphate; NECA, 1-(6-amino-9H-purin-9-yl)-1-deoxy-N-ethyl- $\beta$-D-ribofuranuronamide.

colon were stained with HE or kept in RNA stabilization solution for further analysis.). All tumors from the experimental groups were confirmed as adenomas with high-grade dysplasia compared with the normal intestinal mucosa in the control group, which was consistent with the histopathological score (Fig. 3A). The histopathological evaluation score in the APCP group was significantly lower compared with the NECA group $(\mathrm{P}<0.05$; Fig. 3B). Subsequently, qPCR was performed to detect the gene expression levels of several inflammatory cytokines in colonic tissues, including TNF- $\alpha$, TNF- $\beta$, IL-10 and IL- 6 . The expression of TNF- $\alpha$ and IL- 6 significantly increased in the NECA group compared with the model group, while the differences in the expression of TNF- $\alpha$ and IL-6 between the APCP and model groups was not statistically significant (Fig. 3C and D). There was no significant difference in the expression of TNF- $\beta$ and IL-10 among the four groups (Fig. 3E and F).

Identification of differentially expressed genes (DEGs) and genes associated with CAT by RNA sequencing. To identify genes associated with CAT, RNA sequencing of mice in the model, APCP and NECA groups was conducted. A mean of 23.88 million clean reads were obtained that mapped on the mouse genome with a reliable high mean mapping rate of $93.69 \%$, representing a mean of 20,677 genes that were expressed in each sample. Subsequently, DEGs in the model vs. APCP groups, model vs. NECA groups and NECA vs.
APCP groups were analyzed. Compared with the APCP group, a total of 180 upregulated and 222 downregulated genes were detected in the model group (Fig. S1A). Compared with the NECA group, a total of 113 upregulated and 285 downregulated genes were detected in the model group (Fig. S1B). Compared with the APCP group, a total of 259 upregulated and 89 downregulated genes were detected in the NECA group (Fig. S1C). GO functional annotation of the DEGs indicated that the upregulated and downregulated genes could be classified into 6 major categories, including 'cellular processes', 'environmental information processing', 'genetic information processing', 'human diseases', 'metabolism' and 'organismal systems' (Fig. S1A-C). KEGG pathway analysis indicated the different pathway distributions of DEGs in the model vs. APCP, model vs. NECA and NECA vs. APCP groups (Fig. 4A-C). Among the differentially upregulated and downregulated genes among the three experimental groups, 8 genes were identified as having low expression levels in the APCP group compared with the model group, and high expression levels in the NECA group compared with the model group (Fig. S1D). The 8 genes were as follows: Arachidonate 15-lipoxygenase (ALOX15), carcinoembryonic antigen-related cell adhesion molecule 2 (Ceacam2), mucosal pentraxin 1 (Mptx1), regulating synaptic membrane exocytosis 2 (Rims2), Bcl-2-like protein 15 (Bcl2115), N-acetylaspartate synthetase (Nat81), alpha-defensin 42 (Defa42) and BC037156. Among these 8 genes, ALOX15, Bc12115 and Nat81 were indicated 
A

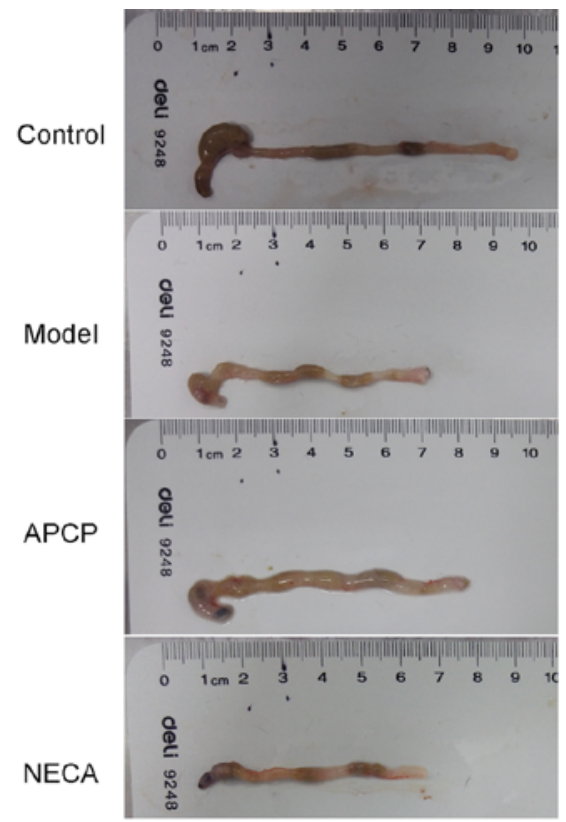

C

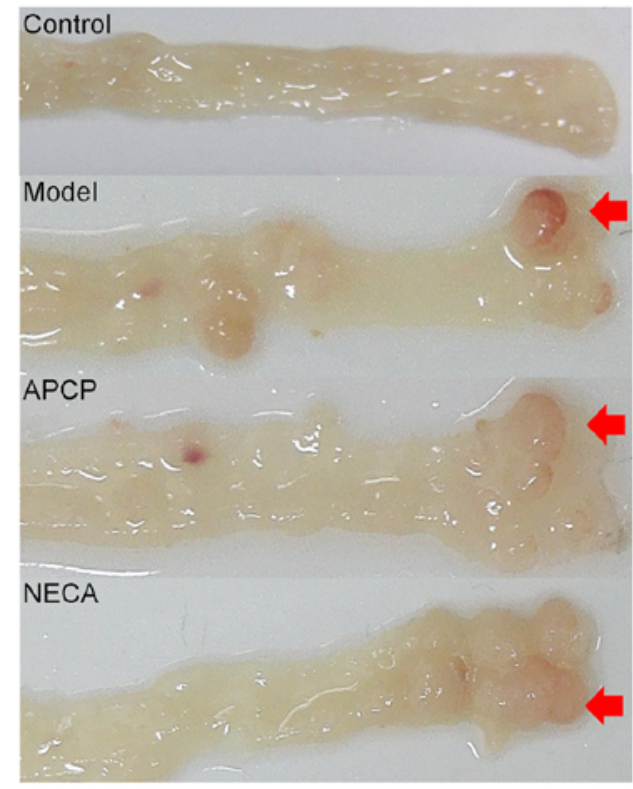

E

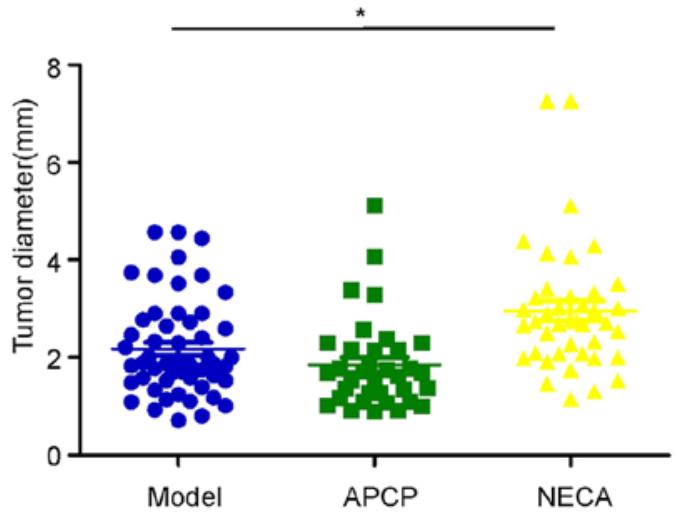

B

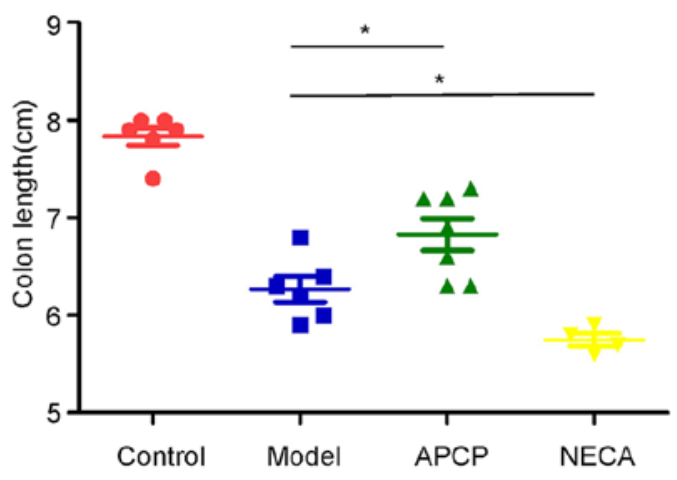

D
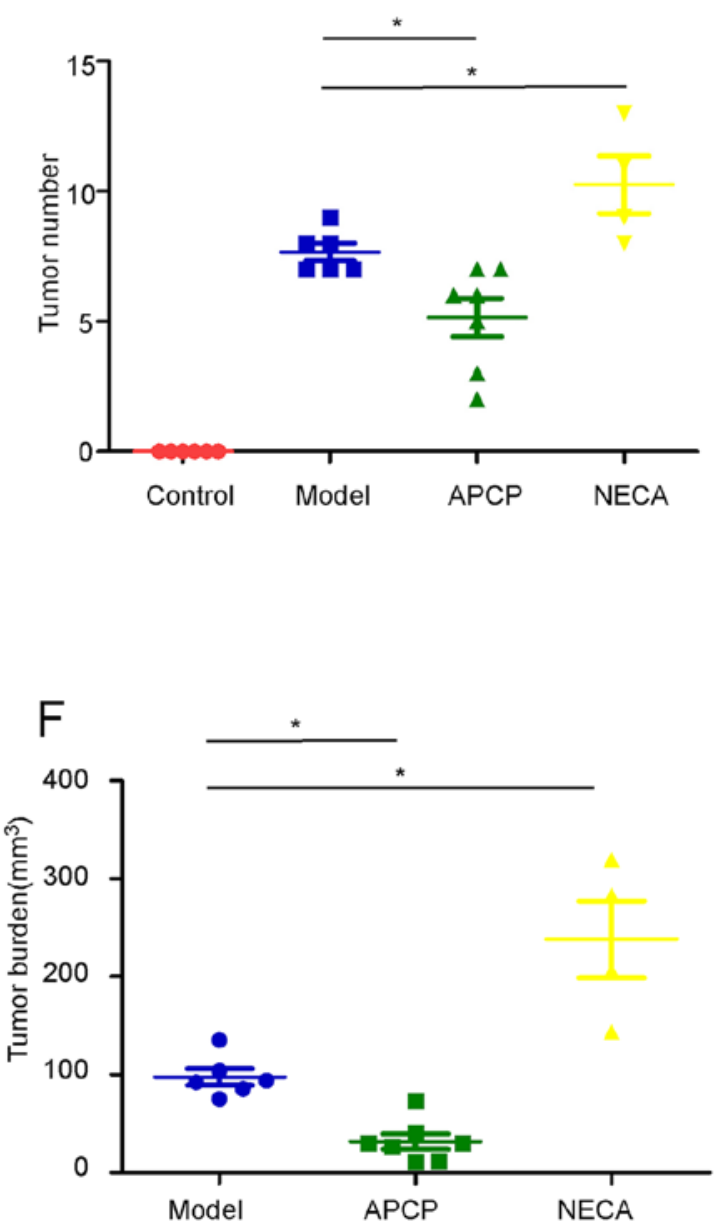

Figure 2. APCP attenuates and NECA aggravates azoxymethane/dextran sulfate sodium-induced colitis-associated tumorigenesis. (A) Representative images of mouse colon length. (B) Comparison of colon length. (C) Macroscopic inspection of mouse colonic tumor number and diameter. Tumors are indicated by red arrows. Comparison of (D) tumor number, (E) diameter and (F) tumor burden. Values are expressed as the mean \pm SEM. ${ }^{*}<0.05$. APCP, adenosine 5'-( $\alpha, \beta$-methylene) diphosphate; NECA, 1-(6-amino-9H-purin-9-yl)-1-deoxy-N-ethyl- $\beta$-D-ribofuranuronamide.

as pathogenesis-associated genes, which were previously reported to play an important role in tumorigenesis (24-26).
The expression levels of these three DEGs were then validated by RT-qPCR, and the results were in accordance with the 

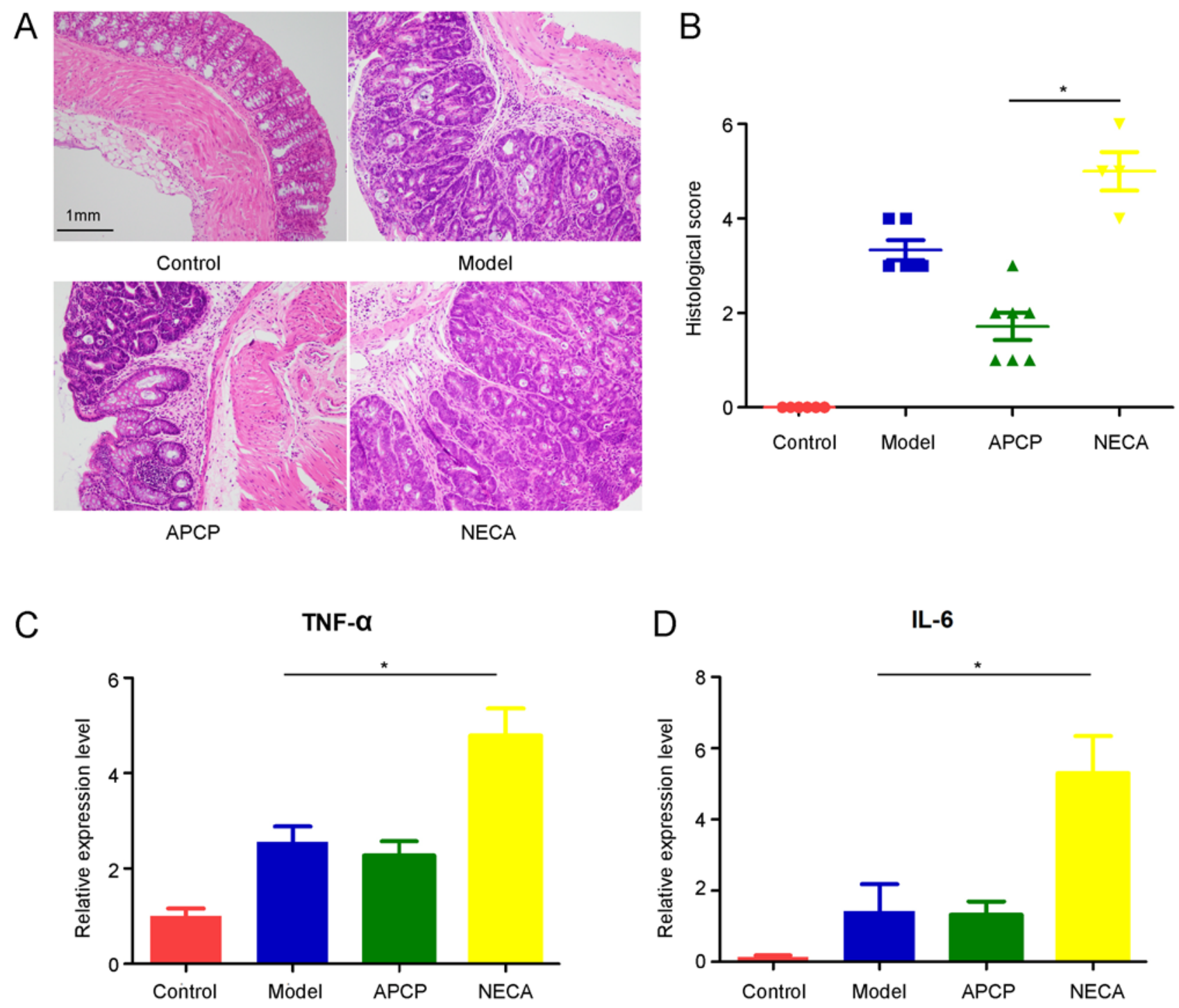

$\mathrm{E}$

TNF- $\beta$

$\mathrm{F}$

IL-10
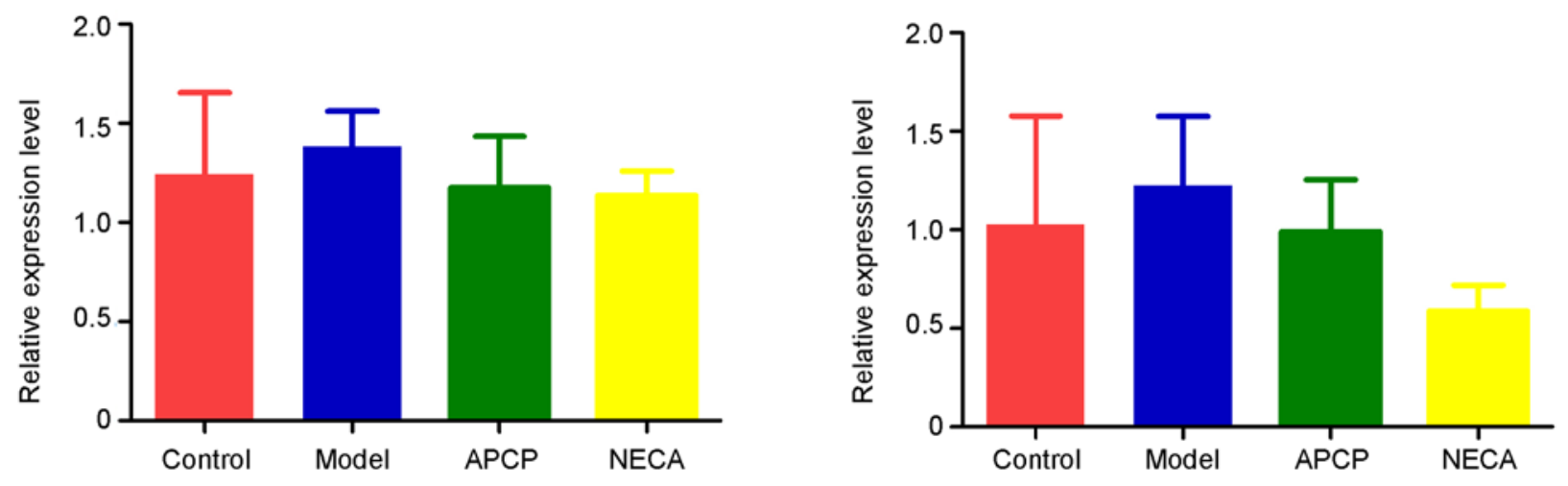

Figure 3. APCP decreases and NECA increases histological damage and expression of inflammatory cytokines in colon tissue. (A) Microscopic examination of colonic tissue by hematoxylin and eosin staining. (B) Comparison of histopathological evaluation score. Quantitative PCR was used to evaluate the expression of inflammatory cytokines (C) TNF- $\alpha$, (D) IL-6, (E) TNF- $\beta$ and (F) IL-10 in colonic tissue. Values are expressed as the mean \pm SEM. "P<0.05. APCP, adenosine 5'-( $\alpha, \beta$-methylene) diphosphate; NECA, 1-(6-amino-9H-purin-9-yl)-1-deoxy-N-ethyl- $\beta$-D-ribofuranuronamide; TNF, tumor necrosis factor; IL, interleukin.

RNA sequencing data. The expression levels of ALOX15, Bcl2115 and Nat8l were higher in the NECA group compared with the APCP and model groups (P<0.05; Fig. 4D). In addition, the expression of ALOX15 was significantly higher in CD73-overexpressing cells compared with their vector-only counterparts (Fig. S2). 
A

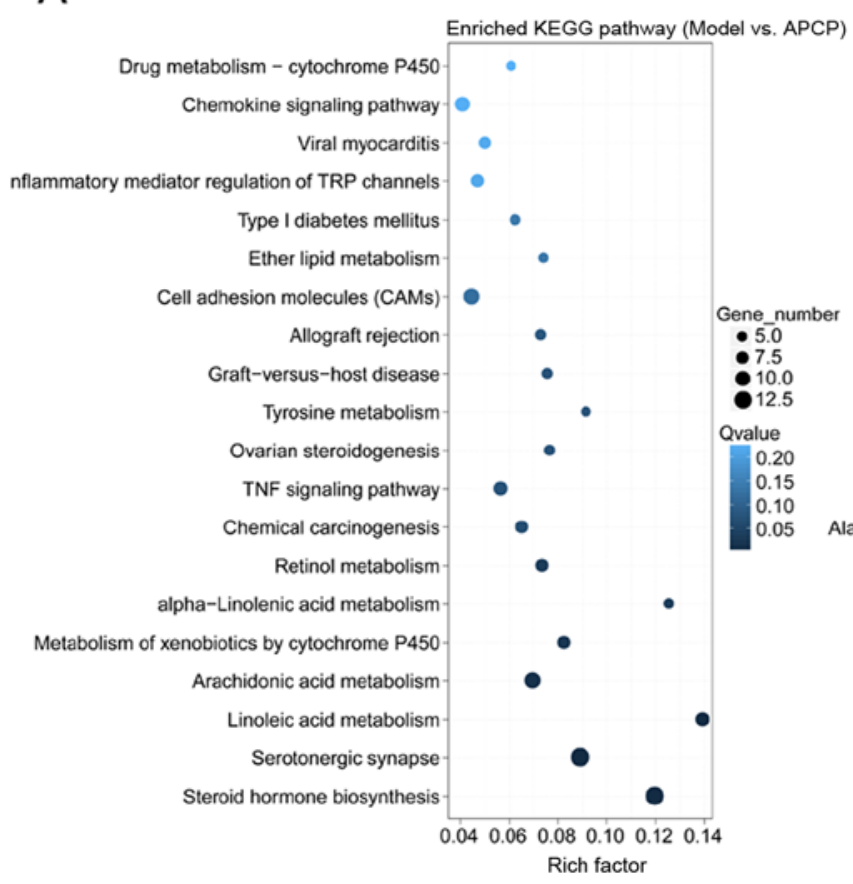

C

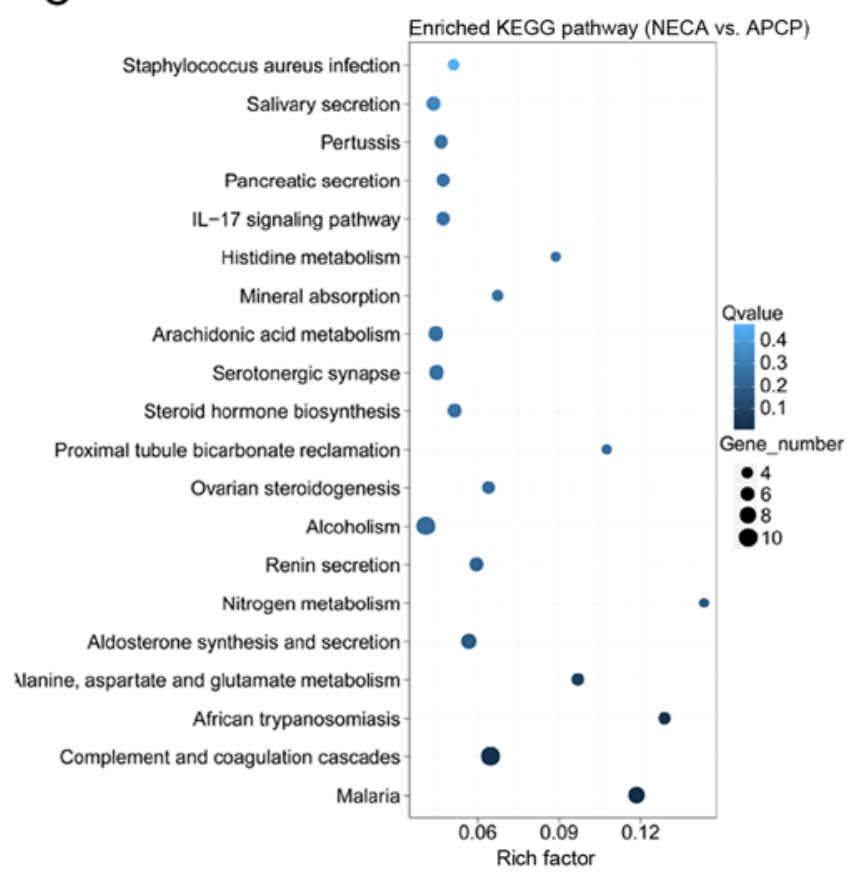

B
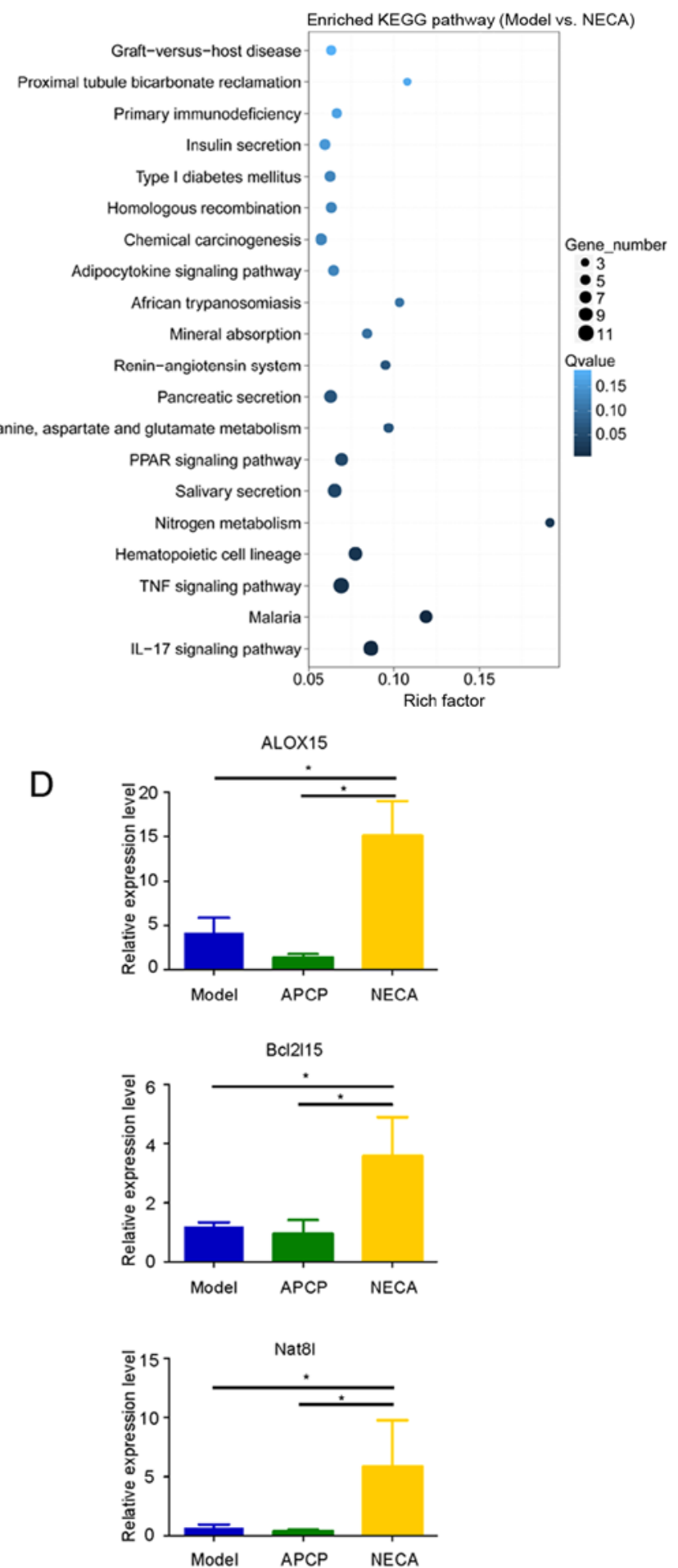

Figure 4. Identification of DEGs and genes associated with colitis-associated tumorigenesis by RNA-sequencing. KEGG pathway analysis indicated the different pathway distributions of the DEGs between (A) model vs. APCP, (B) model vs. NECA and (C) NECA vs. APCP experimental groups. (D) Quantitative PCR was used to validate the expression levels of ALOX15, Bc12115 and Nat81. Values are expressed as the mean \pm SEM. "P<0.05. DEGs, differentially-expressed genes; GO, Gene Ontology; KEGG, Kyoto Encyclopedia of Genes and Genomes; APCP, adenosine 5'-( $\alpha, \beta$-methylene) diphosphate; NECA, 1-(6-amino-9H-purin-9-yl)-1-deoxy-N-ethyl- $\beta$-D-ribofuranuronamide; ALOX15, arachidonate 15-lipoxygenase.

\section{Discussion}

IBD is a multifactorial chronic relapsing disease characterized by an abnormal systemic and local dysregulation of the mucosal immune response $(27,28)$. Previous studies demonstrated that the risk factors for the development of colorectal neoplasia in patients with IBD include disease duration, anatomic extent of the disease, age, family history of CRC and severity of inflammation (29-34). Pro-inflammatory cytokines, such as TNF- $\alpha$, IL-1 $\beta$, IL- 6 and IL- 8 , can promote tumor cell proliferation, thereby promoting carcinogenesis (35-37). 
The primary function of CD73 is to hydrolyze extracellular nucleoside monophosphates, leading to the generation of extracellular adenosine, which is a well-known immunoregulatory metabolite (38). Alam et al (39) observed that CD $73^{-/}$mice were more resistant to infection and exhibited a greater inflammatory response and a significantly lower bacterial load in the liver compared with wild-type mice. By contrast, CD73 expression impaired murine immunity in salmonellosis, leading to increased bacterial colonization and prolonged infection (39). Doherty et al (40) reported that the $\mathrm{CD}^{+} / \mathrm{CD} 4^{+} \mathrm{T}$-cell population in patients with active IBD are enriched with cells with a T-helper 17 (Th17) phenotype, and may be used to monitor disease activity during treatment. These peripheral $\mathrm{CD} 73^{+} / \mathrm{CD} 4^{+} \mathrm{T}$ cells expressed higher levels of pro-inflammatory markers (40). Forte et al (41) observed that inhibition of CD73 by APCP improved B-cell-mediated antitumor immune response in a mouse model of melanoma by affecting both the $\mathrm{CD} 8^{+} \mathrm{T}$-cell- and $\mathrm{B}$-cell-mediated responses.

Given the crucial role of persistent inflammation in the process of CAT and the immunoregulatory abilities of CD73, it was hypothesized that CD73 may play an important role in carcinogenesis in IBD by regulating pathways closely associated with inflammation and immunity. Therefore, the CD73 inhibitor APCP and the adenosine receptor agonist NECA were used in a mouse model of CAT induced by AOM/DSS in immunocompetent C57BL/6J mice in the present study.

The main cause of death in the three experiment groups were due to the severe bloody diarrhea, followed by mucosa inflammatory or intestinal obstruction caused by tumor formation (42). These may reflect the severity of AOM/DSS-induced CAT mouse model, as APCP attenuated while NECA aggravated the survival rate compared with the model group. CD73 was reported to be crucial for the regulation of murine colonic inflammation due to the anti-inflammatory actions of adenosine, mainly through A2 receptor stimulation $(43,44)$. However, other studies using mouse colitis models indicated that inhibiting CD73 may reduce the histological damage of colonic tissue $(39,40)$. In line with these findings, the data of the present study demonstrated that CD73 inhibition attenuated AOM/DSS-induced CAT with reduced histological damage of the colon. It was also observed that tumor number, diameter and tumor burden were significantly increased in the NECA group and decreased in the APCP group compared with the model group. Given the fact that factors affecting tumor progression may lead to changes in the tumor diameter, while those involved in tumor initiation may lead to differences in tumor number, it may be inferred that inhibiting the CD73 pathway may repress both AOM/DSS-induced tumor initiation and progression. Compared with the model group, qPCR demonstrated that the levels of inflammatory cytokines, including TNF- $\alpha$ and IL-6, were significantly increased in the NECA group; however, there were no significant differences in the decreased expression levels of TNF- $\alpha$ and IL- 6 between the APCP and model groups. TNF- $\alpha$ is a pro-inflammatory cytokine secreted by Th17 cells in IBD and IBD-associated cancer $(45,46)$. In addition, IL- 6 is one of the most important pro-inflammatory cytokines mainly produced by myeloid cells and was identified as a key promoter of carcinogenesis (47). However, both NECA and APCP act on all subtypes of the adenosine receptor, thus further studies are required to determine which adenosine receptor subtype is associated with the CAT process. The results of the present CAT mouse model suggested that stimulating the adenosine receptors by NECA might induce immunosuppression and finally lead to tumor immune escape. However, the role of CD73 and adenosine receptors on carcinogenesis induced by inflammation may be different compared with other immunemodulatory effects, not only because it is a complicated process, but because it also depends on the duration that the agonist or inhibitor is administered.

Based on RNA sequencing results, DEGs, such as ALOX15, Bcl2115 and Nat81, were found to be downregulated in the APCP group and upregulated in the NECA group compared with the model group. Namgaladze et al (48) reported that suppressing ALOX15 expression might promote an anti-inflammatory phenol type of IL-4-stimulated human macrophages. Additionally, Kleinstein et al (24) demonstrated that genetic variability in 5-lipoxygenase-activating protein and ALOX15 might affect the risk of colorectal neoplasia, particularly for rectal cancer. Bcl2115 was reported to be involved in CRC cell proliferation (25). Zand et al (26) reported that Nat81 plays a prominent role in promoting tumor growth and represents a valuable target for anticancer therapy. Moreover, a higher Nat8l expression level in colon cancer was associated with worse overall survival (26). Taken together, these studies strongly support the present finding that APCP attenuated while NECA aggravated AOM/DSS-induced CAT in mice. However, the mechanisms through which CD73 regulates ALOX15, Bcl2115 and Nat81, and which of these three genes is the most crucial as a CD73 downstream candidate involved in CAT, warrants further investigation.

In conclusion, the results of the present study suggested that CD73 and its downstream pathway might play an important role in CAT. APCP, an inhibitor of CD73, can inhibit the development of CAT through suppressing the expression of pro-inflammatory cytokines, such as TNF- $\alpha$, IL-6, and downregulating the expression of colorectal tumorigenesis-associated genes, such as ALOX15, Bcl2115 and Nat81.

\section{Acknowledgements}

Part of this study has been presented in the 15th congress of European Crohn's and Colitis Organisation (ECCO), 2020, Vienna/Austria as a meeting text poster abstract.

\section{Funding}

The current study was supported by the National Key R\&D Program of China (grant no. 2017YFC1308800), National Natural Science Foundation of China (grant nos. 81870383 and 81400603), Guangdong Natural Science Foundation (grant no. 2017A030313785), Science and Technology Planning Project of Guangzhou City (grant no. 201804010014), Science and Technology Planning Project of Guangdong Province (grant nos. 2015B020229001 and 20160916), and Clinical Innovation Research Program of Guangzhou Regenerative Medicine and Health Guangdong Laboratory (grant no. 2018GZR0201005). 


\section{Availability of data and materials}

The datasets used and/or analyzed during the present study are available from the corresponding author on reasonable request.

\section{Authors' contributions}

XHL, XRW, PL and XJW contributed to study concept and design. XHL, NL, XBZ, CZ and TH performed the animal model experiments and acquired the data. XHL, XRW, YFC, ZRC, ZXC, PL, XJW participated in the in vitro laboratory experiments and data collection. XRW and XHL contributed to preliminary analysis and interpretation of data and drafting of the manuscript. PL and XJW reviewed the manuscript. All the authors took part in further analysis and revising of the manuscript. XRW, PL and XJW supervised the study. All authors read and approved the final manuscript.

\section{Ethics approval and consent to participate}

The experimental protocol was approved by the Ethics Committee of Sun Yat-sen University (approval no. SYSU -IACUC-2020-B0038). All animal studies were conducted with the approval of the Institutional Animal Care and Use Committee of Sun Yat-sen University.

\section{Patient consent for publication}

Not applicable.

\section{Competing interests}

The authors declare that they have no competing interests.

\section{References}

1. Liu TC and Stappenbeck TS: Genetics and pathogenesis of inflammatory bowel disease. Annu Rev Pathol 11: 127-148, 2016.

2. Khor B, Gardet A and Xavier RJ: Genetics and pathogenesis of inflammatory bowel disease. Nature 474: 307-317, 2011.

3. Grivennikov SI, Greten FR and Karin M: Immunity, inflammation, and cancer. Cell 140: 883-899, 2010.

4. Singh R, Mishra MK and Aggarwal H: Inflammation, immunity, and cancer. Mediators Inflamm 2017: 6027305, 2017.

5. Navaneethan U, Zhu X, Lourdusamy D, Lourdusamy V, Shen B and Kiran R: Colorectal cancer resection rates in patients with inflammatory bowel disease: A population-based study. Gastroenterol Rep (Oxf) 6: 263-269, 2018.

6. Bopanna S, Ananthakrishnan AN, Kedia S, Yajnik V and Ahuja V: Risk of colorectal cancer in Asian patients with ulcerative colitis: A systematic review and meta-analysis. Lancet Gastroenterol Hepatol 2: 269-276, 2017.

7. Lutgens MW, van Oijen MG, van der Heijden GJ, Vleggaar FP, Siersema PD and Oldenburg B: Declining risk of colorectal cancer in inflammatory bowel disease: An updated meta-analysis of population-based cohort studies. Inflamm Bowel Dis 19: 789-799, 2013.

8. Zimmermann H: 5'-Nucleotidase: Molecular structure and functional aspects. Biochem J 285 (Pt 2): 345-365, 1992.

9. Haskó G and Cronstein BN: Adenosine: An endogenous regulator of innate immunity. Trends Immunol 25: 33-39, 2004.

10. Antonioli L, Yegutkin GG, Pacher P, Blandizzi C and Haskó G: Anti-CD73 in cancer immunotherapy: Awakening new opportunities. Trends Cancer 2: 95-109, 2016.

11. Zhang B: CD73: A novel target for cancer immunotherapy. Cancer Res 70: 6407-6411, 2010.
12. Ghalamfarsa G, Kazemi MH, Raoofi Mohseni S, Masjedi A, Hojjat-Farsangi M, Azizi G, Yousefi M and Jadidi-Niaragh F: CD73 as a potential opportunity for cancer immunotherapy. Expert Opin Ther Targets 23: 127-142, 2019.

13. Stagg J, Divisekera U, McLaughlin N, Sharkey J, Pommey S, Denoyer D, Dwyer KM and Smyth MJ: Anti-CD73 antibody therapy inhibits breast tumor growth and metastasis. Proc Natl Acad Sci USA 107: 1547-1552, 2010.

14. Jin D, Fan J, Wang L, Thompson LF, Liu A, Daniel BJ, Shin T, Curiel TJ and Zhang B: CD73 on tumor cells impairs antitumor T-cell responses: A novel mechanism of tumor-induced immune suppression. Cancer Res 70: 2245-2255, 2010.

15. Stagg J, Divisekera U, Duret H, Sparwasser T, Teng MW, Darcy PK and Smyth MJ: CD73-deficient mice have increased antitumor immunity and are resistant to experimental metastasis. Cancer Res 71: 2892-2900, 2011.

16. Wang L, Fan J, Thompson LF, Zhang Y, Shin T, Curiel TJ and Zhang B: CD73 has distinct roles in nonhematopoietic and hematopoietic cells to promote tumor growth in mice. J Clin Invest 121: 2371-2382, 2011.

17. Kaku H, Cheng KF, Al-Abed Y and Rothstein TL: A novel mechanism of B cell-mediated immune suppression through CD73 expression and adenosine production. J Immunol 193: 5904-5913, 2014

18. Grenz A, Zhang H, Eckle T, Mittelbronn M, Wehrmann M, Köhle C, Kloor D, Thompson LF, Osswald H and Eltzschig HK: Protective role of ecto-5'-nucleotidase (CD73) in renal ischemia. J Am Soc Nephrol 18: 833-845, 2007.

19. Eckle T, Krahn T, Grenz A, Köhler D, Mittelbronn M, Ledent C, Jacobson MA, Osswald $H$, Thompson LF, Unertl $K$ and Eltzschig HK: Cardioprotection by ecto-5'-nucleotidase (CD73) and A2B adenosine receptors. Circulation 115: 1581-1590, 2007.

20. Mahamed DA, Toussaint LE and Bynoe MS: CD73-generated adenosine is critical for immune regulation during Toxoplasma gondii infection. Infect Immun 83: 721-729, 2015.

21. Ray MA, Johnston NA, Verhulst S, Trammell RA and Toth LA: Identification of markers for imminent death in mice used in longevity and aging research. J Am Assoc Lab Anim Sci 49: 282-288, 2010.

22. Yang X, Zhang F, Wang Y, Cai M, Wang Q, Guo Q, Li Z and $\mathrm{Hu}$ R: Oroxylin A inhibits colitis-associated carcinogenesis through modulating the IL-6/STAT3 signaling pathway. Inflamm Bowel Dis 19: 1990-2000, 2013.

23. Livak KJ and Schmittgen TD: Analysis of relative gene expression data using real-time quantitative PCR and the 2(-Delta Delta C(T)) method. Methods 25: 402-408, 2001.

24. Kleinstein SE, Heath L, Makar KW, Poole EM, Seufert BL, Slattery ML, Xiao L, Duggan DJ, Hsu L, Curtin K, et al: Genetic variation in the lipoxygenase pathway and risk of colorectal neoplasia. Genes Chromosomes Cancer 52: 437-449, 2013.

25. Xiao R, Li C and Chai B: miRNA-144 suppresses proliferation and migration of colorectal cancer cells through GSPT1. Biomed Pharmacother 74: 138-144, 2015.

26. Zand B, Previs RA, Zacharias NM, Rupaimoole R, Mitamura T, Nagaraja AS, Guindani M, Dalton HJ, Yang L, Baddour J, et al: Role of increased n-acetylaspartate levels in cancer. J Natl Cancer Inst 108: djv426, 2016.

27. Torres J, Mehandru S, Colombel JF and Peyrin-Biroulet L: Crohn's disease. Lancet 389: 1741-1755, 2017.

28. Ungaro R, Mehandru S, Allen PB, Peyrin-Biroulet L and Colombel JF: Ulcerative colitis. Lancet 389: 1756-1770, 2017.

29. Farraye FA, Odze RD, Eaden J and Itzkowitz SH: AGA technical review on the diagnosis and management of colorectal neoplasia in inflammatory bowel disease. Gastroenterology 138: 746-774, 774.e1-4; quiz e12-13, 2010

30. Askling J, Dickman PW, Karlén P, Broström O, Lapidus A, Löfberg $\mathrm{R}$ and Ekbom A: Colorectal cancer rates among first-degree relatives of patients with inflammatory bowel disease: A population-based cohort study. Lancet 357: 262-266, 2001.

31. Fumery M, Dulai PS, Gupta S, Prokop LJ, Ramamoorthy S, Sandborn WJ and Singh S: Incidence, risk factors, and outcomes of colorectal cancer in patients with ulcerative colitis with low-grade dysplasia: A systematic review and meta-analysis. Clin Gastroenterol Hepatol 15: 665-674.e5, 2017.

32. Gyde SN, Prior P, Allan RN, Stevens A, Jewell DP, Truelove SC, Lofberg R, Brostrom O and Hellers G: Colorectal cancer in ulcerative colitis: A cohort study of primary referrals from three centres. Gut 29: 206-217, 1988.

33. Munkholm P, Langholz E, Davidsen M and Binder V: Intestinal cancer risk and mortality in patients with Crohn's disease. Gastroenterology 105: 1716-1723, 1993. 
34. Rogler G: Chronic ulcerative colitis and colorectal cancer. Cancer Lett 345: 235-241, 2014.

35. Popivanova BK, Kitamura K, Wu Y, Kondo T, Kagaya T, Kaneko S, Oshima M, Fujii C and Mukaida N: Blocking TNF-alpha in mice reduces colorectal carcinogenesis associated with chronic colitis. J Clin Invest 118: 560-570, 2008.

36. Azer SA: Overview of molecular pathways in inflammatory bowel disease associated with colorectal cancer development. Eur J Gastroenterol Hepatol 25: 271-281, 2013

37. Taniguchi K and Karin M: IL-6 and related cytokines as the critical lynchpins between inflammation and cancer. Semin Immunol 26: 54-74, 2014.

38. Regateiro FS, Cobbold SP and Waldmann H: CD73 and adenosine generation in the creation of regulatory microenvironments. Clin Exp Immunol 171: 1-7, 2013.

39. Alam MS, Kuo JL,Ernst PB, Derr-Castillo V,Pereira M, Gaines D, Costales M, Bigley E and Williams K: Ecto-5'-nucleotidase (CD73) regulates host inflammatory responses and exacerbates murine salmonellosis. Sci Rep 4: 4486, 2014.

40. Doherty GA, Bai A, Hanidziar D, Longhi MS, Lawlor GO, Putheti P Csizmadia E, Nowak M, Cheifetz AS, Moss AC and Robson SC: CD73 is a phenotypic marker of effector memory Th17 cells in inflammatory bowel disease. Eur J Immunol 42: 3062-3072, 2012.

41. Forte G, Sorrentino R, Montinaro A, Luciano A, Adcock IM, MaiolinoP,ArraC,CicalaC,Pinto A andMorelloS:Inhibition ofCD73 improves B cell-mediated anti-tumor immunity in a mouse model of melanoma. J Immunol 189: 2226-2233, 2012.

42. Zhu Y, Gu L, Li Y, Lin X, Shen H, Cui K, Chen L, Zhou F, Zhao Q Zhang J, et al: miR-148a inhibits colitis and colitis-associated tumorigenesis in mice. Cell Death Differ 24: 2199-2209, 2017.
43. Odashima M, Bamias G, Rivera-Nieves J, Linden J, Nast CC, Moskaluk CA, Marini M, Sugawara K, Kozaiwa K, Otaka M, et al: Activation of A2A adenosine receptor attenuates intestinal inflammation in animal models of inflammatory bowel disease. Gastroenterology 129: 26-33, 2005.

44. Sotnikov I and Louis NA: CD73-dependent regulation of interferon alphaA and interleukin-10 in the inflamed mucosa. ScientificWorldJournal 10: 2167-2180, 2010.

45. Mantzaris GJ: Previous cancer and/or lymphoma in patients with refractory IBD-con: Anti-TNF or conventional immunosuppressive treatment. Dig Dis 32 (Suppl 1): S122-S127, 2014.

46. Bandzar S, Gupta S and Platt MO: Crohn's disease: A review of treatment options and current research. Cell Immunol 286: 45-52, 2013.

47. Yu H, Lee H, Herrmann A, Buettner R and Jove R: Revisiting STAT3 signalling in cancer: New and unexpected biological functions. Nat Rev Cancer 14: 736-746, 2014.

48. Namgaladze D, Snodgrass RG, Angioni C, Grossmann N, Dehne N, Geisslinger G and Brüne B: AMP-activated protein kinase suppresses arachidonate 15-lipoxygenase expression in interleukin 4-polarized human macrophages. J Biol Chem 290: 24484-24494, 2015.

This work is licensed under a Creative Commons Attribution-NonCommercial-NoDerivatives 4.0 International (CC BY-NC-ND 4.0) License. 